Transcripts of CYP26B1 were strongly down regulated $(-12$ to -20 fold, $p<0.01)$ in all three groups based on microarray analysis. RT-PCR revealed the down regulation in all groups, however, significantly only in group $B$, in which the strongest (20-fold) regulation based on the microarray-analysis was found. Also transcripts of retinoid $x$ receptors ( $R X R A, R X R B$ ) were down regulated $(-1.6$ to $-2.0, p<0.05)$ in group $A$ and $C$ based on microarray data. We can report that the gene expression of CYP26B1 and of retinoid ligands is targeted by willow bark, its salicin rich ethanol fraction and imipramine in the peripheral blood of rats, but to a variable extent. Further studies are recommended to fully understand the mode of action of willow bark and its constituents on the retinoid acid metabolism for their further systematic development as co-medication in indications with a prevailing low grade inflammation.

References: (1) Krivospitskaya O et al. Molecular Medicine 2012, March 09. Doi 10.2119. (2) Seldenrijk A et al. J Psychosom Res. 2010 Aug; 69 (2):203-10. (3) Ulrich-Merzenich et al. Phytomedicine 2012, 19 (3-4): 322-9.

\title{
BIOSAFETY ASSESSMENT OF SILYMARIN AND ARGININE COMBINATION IN HEALTHY MEN
}

\section{() Ulrichova Jitka ${ }^{1,}$ Valentova Katerina ${ }^{1}$, Vidlar Ales ${ }^{2}$, Simanek Vilim ${ }^{1}$}

${ }^{1}$ Department of Medical Chemistry and Biochemistry, Faculty of Medicine and Dentistry, Hnevotinska 3, Olomouc, 77515, Czech Republic

${ }^{2}$ Department of Urology, University Hospital, I. P. Pavlova 5, Olomouc, 77500, Czech Republic

Silymarin (SMN) in milk thistle is used as a heptoprotective against alcohol and drug toxicity. Silymarin is also being investigated for its cancer preventive and cholesterol lowering properties (1). L-arginine (L-Arg), a semi-essential amino acid, is recommended as an immune and vitality enhancer (2) but with some urinary side effects (3). This controlled intervention trial aimed to investigate the effect on metabolic parameters of a beverage containing SMN and L-Arg in 20 healthy men aged $41-59$ years. The design of the study is shown in Fig.

ADAPTATION WASHING
\[ \text { ON day 0, } 10 \text { and 27: Blood collections and examination of urodynamic parameters } \]
DIETARY RESTRICTION

Fig. Design of the intervention study.

The subjects drank $500 \mathrm{ml} /$ day beverage without SMN and Arg for 10 days followed by the beverage with $400 \mathrm{mg}$ SMN and $295 \mathrm{mg}$ L-Arg for 10 days. Blood and urine samples were collected on days 0,10 and
27. Total antioxidative capacity, plasma/erythrocyt oxidative stress and clinical chemistry safety markers, urination parameters including IPSS, voiding parameters-rate of urine flow $\left(Q_{\max }\right)$, average flow $\left(Q_{\text {ave }}\right)$, total volume and postvoid residual volume (RV) parameters were measured. The data suggest that the combination of SMN plus L-Arg alters the antioxidant status of erythrocyte, other clinical markers or urinary parameters were unchanged. Overall, the drink was well-tolerated with no adverse effects.

References: (1) Kren V, Walterova D. Silybin and silymarin-new effects and applications. Biomed Pap Med Fac Univ Palacky Olomouc Czech Repub. 2005, 149 (1): 29-41. (2) Bescós R, Sureda A, Tur JA, Pons A. The effect of nitric-oxide-related supplements on human performance. Sports Med. 2012, 42 (2): 99-117. (3) Stothers L, Laher I, Christ GT. A review of the L-arginine - nitric oxide - guanylate cyclase pathway as a mediator of lower urinary tract physiology and symptoms. Can J Urol. 2003, 10 (5): 1971-80. 